\title{
Cenobamate: New Antiseizure Medication with High Seizure Freedom and Dual Mechanisms of Action
}

\author{
Steve S. Chung* MD \\ Department of Neurology, Banner University Medical Center, University of Arizona, Phoenix, Arizona, USA
}

${ }^{\star}$ Corresponding author: Steve S. Chung, MD, Department of Neurology, Banner University Medical Center, University of Arizona, 755 E. McDowell Road, Phoenix, Arizona 85006, USA; Tel: 602-521-3234; Email: steve.chung@bannerhealth.com

Received: May 06, 2021; Accepted: May 26, 2021; Published: July 07, 2021

\begin{abstract}
BCenobamate is a newly approved antiseizure medication for patients with focal epilepsy. Even though preclinical study revealed potential for both generalized and focal epilepsy, clinical study has been completed in refractory focal epilepsy patients to date. The efficacy of cenobamate at 100 to 400 $\mathrm{mg}$ per day has been strongly supported by two pivotal studies with significant seizure reduction and approximately $20 \%$ of seizure freedom rate among the refractory epilepsy patients. Cenobamate was well tolerated and the most frequently reported adverse effects during studies were somnolence and dizziness. In order to mitigate the potential hypersensitivity drug rash with eosinophilia and systemic symptoms (DRESS), cenobamate should be started at a low dosage of $12.5 \mathrm{mg}$ per day and titrated at 2-week intervals. Cenobamate has dual mechanisms of action of inhibiting excitatory sodium channels and enhancing inhibitory GABAA currents as an allostatic modulator.
\end{abstract}

\section{Introduction}

Epilepsy is one of the most common neurological disorders affecting up to two percent of the population worldwide, and more than 3.5 million people in the United States alone. Treatment of epilepsy often imposes an exposure to various antiseizure medications (ASMs) and requires long-term commitment and compliance from the patient. Excluding the small percentage of people who underwent successful epilepsy surgery, large portion of patients are maintained through chronic medical management for seizure control. Despite the advent of new antiseizure medications (ASMs) over the past 25 years, approximately $30 \%$ of epilepsy patients experience recurrent seizures and many experience undesirable side effects. Cenobamate (also known as YKP3089, Xcopri') is a new ASM, that received US Food and Drug Administration (FDA) approval for the treatment of focal onset seizures in adults as both monotherapy and adjunctive therapy in November 2019 [1]. Cenobamate is a novel tetrazole alkyl carbamate derivative, (1R)-1-(2-chlorophenyl)-2-(tetrazol-2-yl) ethyl carbamate. Pivotal clinical trials reported significant seizure improvement and favorable tolerability profile in patients with focal epilepsy.

\section{Pharmacokinetics}

Cenobamate was well absorbed after oral ingestion with $\geq 88 \%$ recovered in the urine. Fasting/fed studies showed no influence of food on the rate or extent of cenobamate absorption [2]. Plasma protein binding of cenobamate (mostly albumin) is about $60 \%$ in healthy subjects. The half-life for cenobamate is approximately 50 to 60 hours after single doses of 100 to $400 \mathrm{mg}$. Mild to moderate renal impairment may increase cenobamate plasma concentrations by 1.5-fold, while mild to moderate hepatic impairment may increase cenobamate plasma concentrations by 2 -fold [2].

\section{Drug Interactions}

In dedicated drug interaction studies, changes in cenobamate exposures and plasma concentrations with other AEDs were generally less than $20 \%$. Cenobamate was reduced $28 \%$ by phenytoin due to hepatic enzyme induction of phenytoin. On the other hand, due to CYP 2C19 inhibition of cenobamate, phenytoin exposure (AUC) can be increased by 84\% and phenobarbital AUC by 37\%. For the same reason, cenobamate can increase omeprazole and escitalopram exposure by 2 -fold. In addition, cenobamate induces CYP3A4/5 which may reduce exposure to other medications such as midazolam, alprazolam, oral contraceptive pills, and simvastatin. Cenobamate also induces CYP2B6 and medications such as bupropion dosage should be increased as needed [3].

\section{Mechanisms of Action}

Cenobamatehasabroad-spectrum ofanticonvulsanteffectsin several animal models, including maximal electroshock model (traditional focal seizure model), pentylenetetrazol (traditional generalized seizure model), and picrotoxin models as well as hippocampal kindled and 6 $\mathrm{Hz}$ stimulation models. Cenobamate inhibits voltage gated sodium channels and enhances $\mathrm{GABA}_{\mathrm{A}}$ receptors in rodents. Cenobamate's effects on persisting sodium channel currents are different from other sodium channel blocking ASMs. Cenobamate is also a positive allosteric modulator of $\mathrm{GABA}_{\mathrm{A}}$ receptors in hippocampal neurons, and binds at a non-benzodiazepine site and enhances $\mathrm{GABA}_{\mathrm{A}}$ receptor-mediated inhibitory currents. however, anti-seizure mechanisms in humans have not been fully elucidated.

\section{Efficacy}

The efficacy of cenobamate was established by the results from two double-blind, randomized, placebo-controlled, parallel-group, 
multicenter, multinational studies (Studies C013 and C017). Both studies were performed in adult patients with medically refractory focal epilepsy to evaluate the efficacy, safety, and tolerability of cenobamate. In addition, one open label safety study (C021) was completed. Both $\mathrm{C} 013$ and $\mathrm{C} 017$ studies required to have at least 3-4 focal seizures per 28 days with stable doses of 1-3 ASMs. Patients had a mean duration of epilepsy of approximately 22 years and median baseline seizure frequency of 8.5 seizures per 28 days. Study C013 enrolled 113 patients to the single target dose of $200 \mathrm{mg} /$ day cenobamate arm, and 109 adult patients to placebo. A statistically significant improvement was observed in the 28-day median percent seizure reductions from baseline, with a reduction of $55.6 \%(p<0.0001)$ in the cenobamate $200 \mathrm{mg}$ /day group, compared to $21.5 \%$ in the placebo arm [4]. In addition, a significant difference in the $50 \%$ responder rates (patients with $\geq 50 \%$ reduction in seizure frequency from baseline to maintenance) were also observed in the cenobamate treatment group $(50 \%, p<0.0001)$, compared to placebo (22\%). Seizure freedom rate $(100 \%$ seizure reduction during the maintenance period) was $28 \%$ ( $p=0.0001)$ among cenobamate group, compared to $1 \%$ for placebo (Figure 1). Study C017 was a blinded, randomized treatment trial comparing three cenobamate doses vs. placebo; subjects were randomly assigned to cenobamate $100 \mathrm{mg}$ (n $=108), 200 \mathrm{mg}(\mathrm{n}=110)$, or $400 \mathrm{mg}(\mathrm{n}=111)$ per day and placebo $(n=108)$. The median percent reductions from baseline in seizure frequency per 28 days were $36.3 \%, 55.2 \%$, and $55.3 \%$ for those patients who received cenobamate at $100 \mathrm{mg}, 200 \mathrm{mg}, 400 \mathrm{mg}$, respectively, compare to $24.3 \%$ in placebo arm [5]. The secondary end point, $50 \%$ responder rates, during the maintenance phase were significantly higher for the cenobamate $100 \mathrm{mg}(40 \%, p=0.0365), 200 \mathrm{mg} /$ day $(56 \%, p<0.0001)$, and $400 \mathrm{mg} /$ day $(64 \%, p<0.0001)$ compared to placebo (25\%). Seizure freedom rate during the 12 -week maintenance phase were $4 \%(p=0.3688)$ for the cenobamate $100 \mathrm{mg}$ group, $11 \%$ $(p=0.0022)$ for the cenobamate $200 \mathrm{mg}$ group, and $21 \%(p<0.0001)$ for the cenobamate $400 \mathrm{mg}$ group (Figure 2 ). In addition, cenobamate showed statistically significant reductions in seizure frequency across all types of focal seizures including focal aware motor, focal impaired awareness, focal to bilateral tonic-clonic seizures at doses of 100, 200, and $400 \mathrm{mg}$ /day in both studies.

\section{Study C013: Reduction in Seizure Frequency from Baseline (\%)}

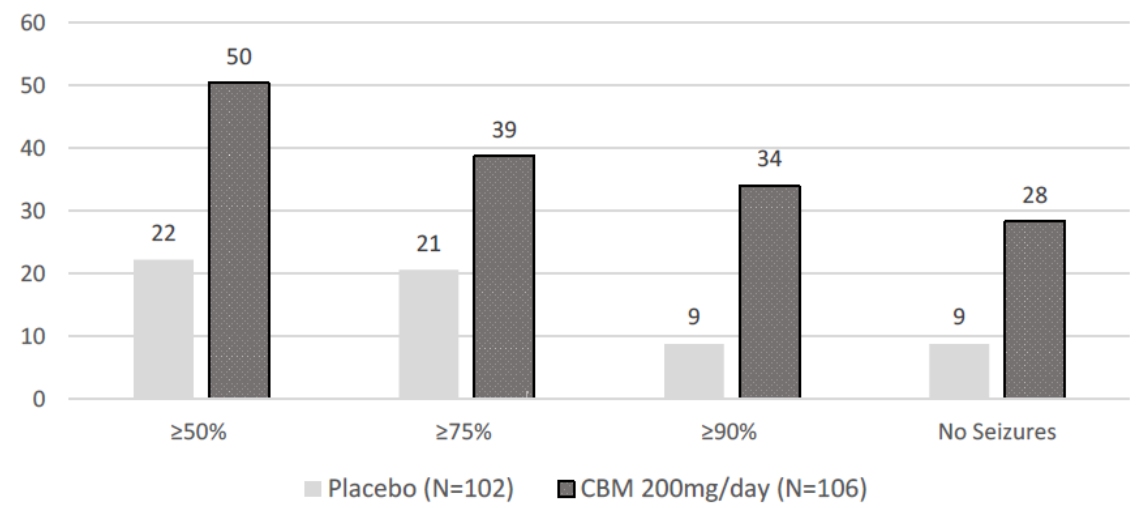

$\mathrm{CBM}=$ cenobamate

Figure 1: Proportion of Patients Exhibiting Different Percent Seizure Reductions During the Maintenance Phase over Baseline in Study C013.

Study C017: Reduction in Seizure Frequency from Baseline (\%)

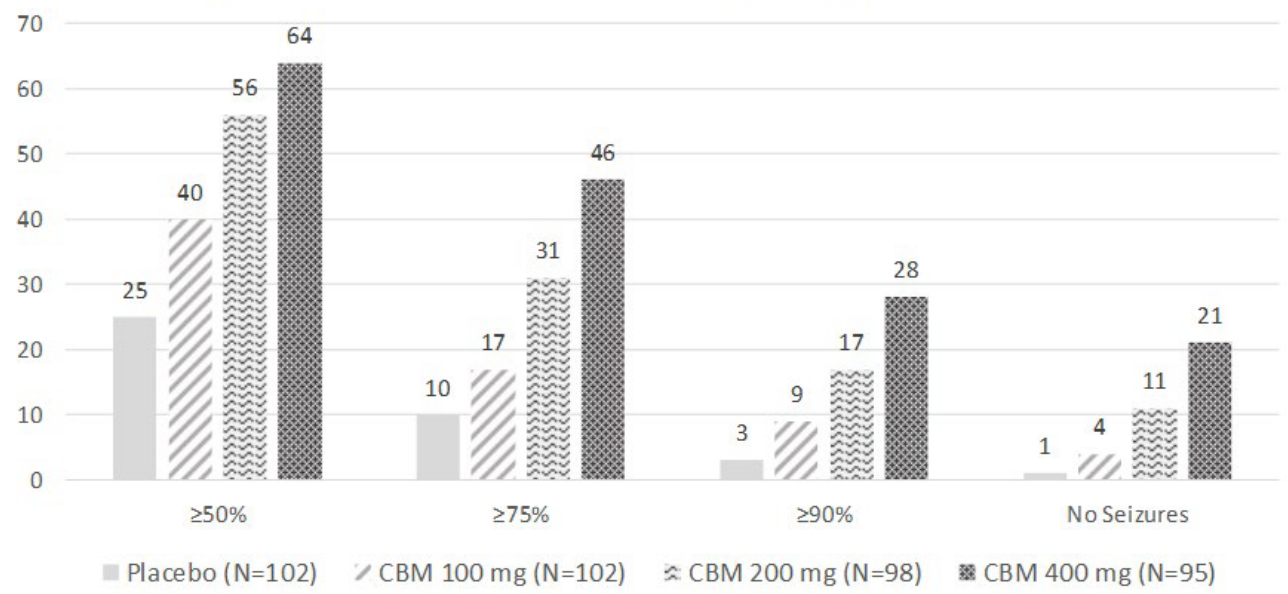

$\mathrm{CBM}=$ cenobamate

Figure 2: Proportion of Patients Exhibiting Different Percent Seizure Reductions During the Maintenance Phase over Baseline in Study C017. 


\section{Safety and Tolerability}

The most frequent Treatment-Emergent Adverse Effects (TEAEs) reported with cenobamate were dose-dependent increases in somnolence and fatigue-related adverse reactions. In both placebocontrolled trials, $31 \%$ of patients randomized to receive cenobamate at $100 \mathrm{mg} /$ day, $36 \%$ at cenobamate $200 \mathrm{mg} /$ day, and $57 \%$ of cenobamate at $400 \mathrm{mg} /$ day reported at least one of these, compared to $19 \%$ of patients who received placebo [4,5]. The second most common cenobamate related TEAEs were dizziness and disturbance in gait and coordination. Cognitive dysfunction related events (i.e., memory impairment, disturbance in attention, amnesia, confusion, aphasia, speech disorder, slowness of thought, disorientation, and psychomotor retardation) were low and occurred in $6 \%$ of cenobamate patients at $100 \mathrm{mg} /$ day, $6 \%$ of cenobamate at $200 \mathrm{mg} /$ day, and $9 \%$ of cenobamate at $400 \mathrm{mg} /$ day, compared to $2 \%$ of placebo patients. The adverse reactions leading to discontinuation, in descending order of frequency, were ataxia, dizziness, somnolence, diplopia, nystagmus, and vertigo $[4,5]$. In a placebo-controlled study, cenobamate had higher rate of QT shortening ( $31 \%$ at $200 \mathrm{mg}$ and $66 \%$ at $500 \mathrm{mg}$ ) compared to placebo (6-17\%) [1]. Reductions of the QTc interval below $300 \mathrm{msec}$ were not observed with cenobamate, but the patients with Familial Short QT syndrome should not be treated with cenobamate. The incidence of skin rash was not higher in cenobamate-treated patients than with placebo-treatment, with only 2 cases reported $(0.45 \%)$. However, three subjects (one epilepsy patient and two healthy volunteers) developed drug rash with eosinophilia and systemic symptoms (DRESS) among 2,528 study patients. In all three cases, cenobamate was initiated at a higher starting dose and titrated faster. After these cases, C021 study $(\mathrm{n}=1,339)$ was conducted at the lower starting dose with slower titration rate. Subjects in this study began cenobamate at a low starting dose of $12.5 \mathrm{mg} /$ day and titrated up slowly every 2 weeks to a target dose of $200 \mathrm{mg} /$ day and then up to a possible maximum dose of $400 \mathrm{mg} /$ day as tolerated. With this slow initiation and titration of cenobamate, no cases of DRESS were reported [6].

\section{Dosing}

To mitigate the risk for DRESS, cenobamate should be titrated slowly with the starting dose of $12.5 \mathrm{mg} /$ day and titrated at 2-week intervals to a maintenance dose of $200 \mathrm{mg} /$ day, with additional titration to a maximum dose of $400 \mathrm{mg} /$ day as needed (Table 1).
Table 1: Recommended cenobamate titration at 2-week intervals.

\begin{tabular}{|c|c|}
\hline Weeks $1-2$ & $12.5 \mathrm{mg} /$ day \\
\hline Weeks $3-4$ & $25 \mathrm{mg} /$ day \\
\hline Weeks $5-6$ & $50 \mathrm{mg} /$ day \\
\hline Weeks $7-8$ & $100 \mathrm{mg} /$ day \\
\hline Weeks $9-10$ & $150 \mathrm{mg} /$ day \\
\hline Weeks 11 and maintenance & $200 \mathrm{mg} /$ day \\
\hline
\end{tabular}

\section{Key Points and Conclusion}

Cenobamate is a newly approved ASM for the treatment of focal epilepsy in adults. In three clinical studies, cenobamate showed significant seizure improvement at 100-400 mg once daily with a high seizure freedom rate even in refractory epilepsy population. Due to potential idiosyncratic DRESS, cenobamate should be started at 12.5 mg per day with gradual increase every two weeks. Cenobamate has dual mechanisms of action of inhibiting excitatory sodium channels and enhancing $\mathrm{GABA}_{\mathrm{A}}$ inhibitory currents. It is important to note that due to hepatic enzyme CYP3A4/5 induction and CYP2C19 inhibition, certain medication exposure can be affected by coadministration of cenobamate. Most commonly reported adverse effects of cenobamate were somnolence and dizziness, which were usually reported as mild to moderate. In conclusion, cenobamate expands treatment options for patients with focal epilepsy and may provide significant benefit to those patients who remain refractory to other AEDs.

\section{References}

1. Xcopri. Prescribing information. Paramus, NJ: SK Life Science; 2019.

2. Vernillet L, Greene SA, Kamin M (2020) Pharmacokinetics of Cenobamate: Results From Single and Multiple Oral Ascending-Dose Studies in Healthy Subjects. Clinical Pharmacology in Drug Development. 9: 428-443. [crossref]

3. Greene SA, Kwak C, Kamin M, et al. (2019) The Effect Of Cenobamate On The Single-Dose Pharmacokinetics Of Multiple Cytochrome P450 Probes Using A Cocktail Approach In Healthy Subjects [Data on File]. SK Life Science.

4. Chung SS, French JA, Kowalski J, Krauss GL, Lee SK, Maciejowski M, et al. (2020) Randomized phase 2 study of adjunctive cenobamate in patients with uncontrolled focal seizures. Neurology 94 . [crossref]

5. Krauss GL, Klein P, Brandt C, Lee SK, Milanov I, et al. (2020) Safety and efficacy of adjunctive cenobamate (YKP3089) in patients with uncontrolled focal seizures: a multicentre, double-blind, randomised, placebo-controlled, dose-response trial. Lancet Neurol 19: 38-48.

6. Sperling MR, Klein P, Aboumatar S, Gelfand M, Halford JJ, et al. (2020) Cenobamate (YKP3089) as adjunctive treatment for uncontrolled focal seizures in a large, phase 3 multicenter, open-label safety study. Epilepsia 6: 1099-1108. [crossref] 